\title{
Effect of coordination exercise on improving some locomotor and physical abilities and ease attention deficit hyperactivity disorder within handicapped learnable children
}

Dr. / Eslam Mohamed Mahmoud Salem ${ }^{(*)}$

Introduction:

Handicapped children have the right to live, development and education to be productive, like other society members. Mental disabilities have special nature and are not similar to any other disability due to its multiple aspects and the fact that society progress depends on mental competency of its members.

It is necessary to care with mentally retarded handicapped children ,work on investment their potentials, and ensure their adjustment with environment around them. It is also important to prepare them to integrate with community members, as well as giving attention to their own development, developing programs to suit their disability nature as basic human right for them and basic guide in raising these children and protect them from social isolation and inactivity life. (Nawasra, 2010, p.158, Special education general management, basic education general management, high education sector, Ministry of Education, 2003, p.34)

Therefore, physical education programs for this category are not different than others for normal pupils, but disability slow learning process; as well as that mentally handicapped pupils have weakness body mechanical performance, so attention should be given to physical and skill activities programs, as it help growth of motor, social, and psychological skills. (Nawasra, 2010, p .158)

Mentally disabled child cannot acquire a skill in same degree as normal children; it should be emphasized on learning basic motor skills such as right standing, walking, running, jump, as basic important movements to adapt to environmental. It don't require much cognitive aspects or high coordination between body parts; in same time it work ti increase locomotor and physical abilities level to improve muscle tone. It is highly possible that mentally disabled children get impaired physical and motor fitness compared to ordinary peers as a result of avoiding participation in motor activities. (Alhagrasy, 2002, p .220, Macintyre, 2002, p.117, Nawasra, 2010, p. 159).

Attention deficit hyperactivity disorder (ADHD) is of serious behavioral disorders in mental health field; which spread among ordinary children generally, and mentally handicapped in

\footnotetext{
${ }^{(*)}$ Assistant professor, gymnastic and exercises training department, faculty of physical education for men, Alexandria university
} 
particular. It has negative effect on these children, and affect functionality, they face difficulty in self-control, and it impedes them from acquisition of basic skills necessary to adapt to environment (Abdullah, 2003, p.117, Aldesouqy, 2006, p.22, and Alsharif, 2014, p. 27).

ADHD is about children, adolescents and adults who exhibit behavioral patterns like lack attention, impulsivity, hyperactivity, where the child is permanently active to be subject of a complaint from others surrounding him (Aldesouqy, 2006, p.17, and Alqady, 2011, p. 23).

ADHD is a behavioral neurological disorder caused by brainimpaired function, affecting behavior, thoughts and emotions, and can be dealt with and alleviate its symptoms in order to help the child to learn and self-control (Mohammad, 2010, p .105).

ADHD symptoms appear in three types, first dominated by attention deficit symptoms more than hyperactivity and impulsivity, second dominated by both hyperactivity and impulsivity symptoms than attention deficit, and the third combines symptoms of attention deficit, hyperactivity and impulsivity together (Alkhashrany \& EsSayed, 2009, p. 82, and Alsharif, 2014, p.28)

Diagnostic and statistical manual of mental disorders (DSM) indicates that prevalence of this disorder among children of schoolage range between ( 3 and $7 \%$ ), and the available data about ratio between adolescents and adults is very limited, and this percentage increases because of combining the disorder patterns (American Psychiatric Association, 2000, p .7).

ADHD is diagnosed through (personal interview with parents and child to know his behavior at home and school, the application of a set of standards which estimate behavior, obtain data from the school about the child's behavior and achievement tests marks, conducting a series of psychological tests and conducting medical examinations) (Aldesouqy, 2006, p.67, and Barkley, 2003, p .77-83).

While developing activity programs for this category, it should be based on what child acquire from basic motor skills; and give attention to big muscles coordination. Activity should characterized by speed and agility; any activity should take short time due to poor endurance, as well as giving interest to climbing, hung up, jump and running exercises and chase games, and train children on social and democratic relations through good example and direct guidance(Ibrahim, 2005, p .174 and Alsharif, 2014, p. 60).

Most of daily activities require coordination element, especially motor performance activities that use more than one body part in 
more than one direction at same time, coordination depend on right coherence and complementarity between muscle and nervous systems to produce movement accurately and timely(Moghazi, 2010, p. 35)

Coordination is the individual's ability to coordinate movements different in shape and direction accurately and smoothly in one motor performance model (Abdulfatah, 1997, p14. and Abdul Khaliq, 2005, p.169). Coordination is associated with other physical abilities such as speed, agility and accuracy; it associates with speed in motor performance requirements in terms of time, and with agility and accuracy through spatial performance and moving the body and its parts with required accuracy (Abbas, 2005, p .205 and Ibrahim, 2007, p. 16).

Coordination can be developed through various exercises graduated in difficulty. Motor coordination cannot be developed and mastered correctly except after continuous repetitions so that nervous system be able to send mutual nerve signals between cessation and excitement for more than a muscle at the same time in different body parts (Essawi, 1999, p.35; Salama, 2000, p.17; Mahmoud \& Qandil, 2005, p .16, and Ibrahim, 2007, p. 17).

Coordination is divided to nervous coordination (coupling between neural processes leading to solution of kinetic duties), muscle coordination (coordination between tension and relax of muscles to perform the movement), and motor coordination (coordination among body parties)( Abbas, 2005, p .25).

Researcher, through visits to intellectual education schools in Alexandria, found that ADHD is of the most important psychological and behavioral problems within children in general, and handicapped in particular, they represent a source of concern for school and the community. After reviewing motor programs offered to them, the researcher found that programs used are behavioral and guidance program to ease the disorder. They have no motor activity programs developed specially for them, but they use general motor activities and programs. Therefore, researcher thought that it is possible to use coordination exercises to improve locomotor and physical abilities and ease ADHD symptoms within handicapped children.

\section{Research objective}

Identify the effect of coordination exercise on improving some locomotor and physical abilities and ease attention deficit hyperactivity disorder (ADHD) within handicapped learnable children

Research Hypotheses: 
- There are statistically significant differences between pre and post measurements of some locomotor and physical abilities within experimental group handicapped children in favor of post measurements.

- There are statistically significant differences between post measurements of some locomotor and physical abilities within experimental and control group handicapped children in favor of experimental group.

\section{Research procedures:}

\section{Research Methodology:}

Experimental approach used due to its appropriateness of research nature

\section{Human domain:}

This study performed on a sample of mentally handicapped pupils aged ( 9 and12) years; mental age between ( 8 and10) years and IQ score between (55 and 75).

\section{Time domain:}

Pilot study and pre measurements carried out in the period from $26 / 09 / 2015$ to $08 / 10 / 2015$, main experiment carried out in the period from $10 / 10 / 2015$ to $16 / 15 / 2015$, and post measurements in the period from 19/12/2015 to $23 / 12 / 2015$.

\section{Spatial domain:}

Saad Zaghloul and Ikhlas intellectual schools, west educational directorate, Alexandria.

\section{Research sample:}

Main study sample selected intentionally and consisted of (24) mentally disabled students in Sadat school, divided into two equal groups each of (12) pupils, one experimental and one. Pilot study sample consisted of (12) mentally handicapped students.

\section{Pilot studies:}

First pilot study:

Aimed at:

- Determine appropriate tests to measure IQ, physical and locomotor variables, and ADHD within handicapped children.

This study has resulted in:

- Define IQ measure: Godanf test. (Alsharif, 2014, p. 27 and ALhussainy, 2010)

- Determine the physical and locomotor tests (Appendix 1)

- Selecting ADHD checklist from DSM IV, amended by Ahmed Alsharif, 2014, it made up of three aspects containing (32) phrases on quintuple response scale (appendix 2).

\section{Second pilot study:}

Aimed at ensure validity and reliability of research tools. 
I-Validity:

Comparing highest and lowest quartiles used to ensure validity y applying tests on (12) pupils.

Table (1) Significance of differences between highest and the lowest quartiles in locomotor and physical tests

\begin{tabular}{|c|c|c|c|c|c|c|c|c|}
\hline \multirow{2}{*}{\multicolumn{3}{|c|}{ physical variables }} & \multicolumn{2}{|c|}{$\begin{array}{l}\text { Highest } \\
\text { Quartile }\end{array}$} & \multicolumn{2}{|c|}{$\begin{array}{l}\text { Lowest } \\
\text { Quartile }\end{array}$} & \multirow[t]{2}{*}{$\begin{array}{l}\text { Mean } \\
\text { difference }\end{array}$} & \\
\hline & & & Mean & $\pm S D$ & Mean & $\pm S D$ & & \\
\hline Flexibility & & $\begin{array}{l}\text { Trunk front } \\
\text { flexion } \\
\text { from long } \\
\text { sitting test } \\
\text { (cm) }\end{array}$ & $\begin{array}{l}- \\
10.25\end{array}$ & 2.92 & $\begin{array}{l}- \\
5.25\end{array}$ & 1.96 & -5.00 & \\
\hline speed & & $\begin{array}{l}20 \mathrm{~m} \\
\text { running } \\
\text { (second) }\end{array}$ & 7.41 & 0.85 & 5.42 & 0.96 & 1.99 & \\
\hline agility & & $\begin{array}{l}\text { Zigzag } \\
\text { running } \\
\text { (seconds) }\end{array}$ & 19.36 & 0.96 & 17.52 & 1.03 & 1.84 & \\
\hline coordination & \begin{tabular}{|l|} 
Eye-hand \\
coordination
\end{tabular} & $\begin{array}{l}\text { Ball throw } \\
\text { and } \\
\text { receive } \\
\text { within } 30 \\
\text { seconds } \\
\text { (number) }\end{array}$ & 4.27 & 2.01 & 2.15 & 1.36 & 2.12 & \\
\hline & \begin{tabular}{|l|} 
Eye-legs \\
coordination
\end{tabular} & $\begin{array}{l}\text { Numbered } \\
\text { circles test } \\
\text { (seconds) }\end{array}$ & 12.43 & 0.89 & 8.71 & 0.72 & 3.72 & \\
\hline Balance & $\begin{array}{l}\text { Stability } \\
\text { balance }\end{array}$ & $\begin{array}{l}\text { Standing } \\
\text { with feet } \\
\text { on bar } \\
\text { (seconds) }\end{array}$ & 23.21 & 1.95 & 17.36 & 1.83 & 5.85 & \\
\hline & $\begin{array}{l}\text { Moving } \\
\text { balance }\end{array}$ & $\begin{array}{l}\text { walking on } \\
\text { Swedish } \\
\text { bench } \\
\text { (seconds) }\end{array}$ & 9.15 & 0.74 & 5.62 & 0.84 & 3.35 & \\
\hline Accuracy & & $\begin{array}{l}\text { Shooting } \\
\text { on } \\
\text { overlapped } \\
\text { rectangles }\end{array}$ & 4.17 & 0.86 & 3.31 & 0.76 & 0.86 & \\
\hline Locomotor & Time sense & rror 10 & 5.36 & & 3.62 & 0.96 & 1.74 & \\
\hline
\end{tabular}




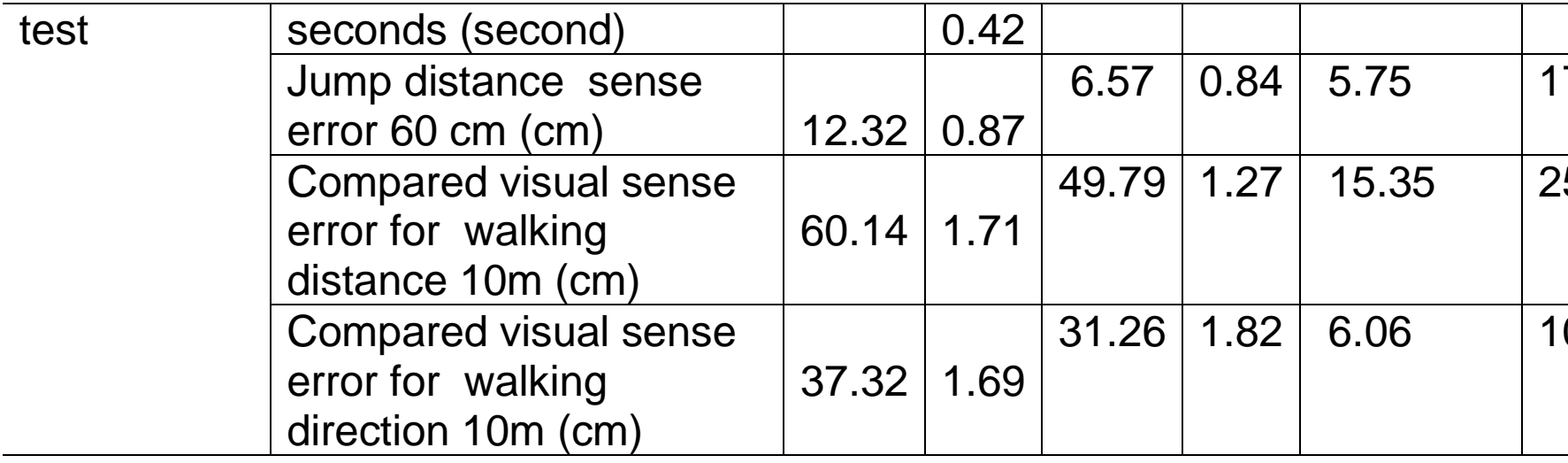

${ }^{*}$ Significant at the 0.05 level $=2.23$

Table (1) results reveal significant differences between

highest and lowest quartiles as $T$ value ranges between $(6.764$

and 25.774) values which is greater than T significant at 0.05 level.

Validity coefficient ranged between $(0.805$ and 0.983$)$, the matter which proof validity of physical and locomotor tests

Table (2) Significance of differences between highest and the lowest quartiles in ADHD checklist for learnable mentally disabled children

\begin{tabular}{|c|c|c|c|c|c|c|c|}
\hline \multirow{2}{*}{$\begin{array}{l}\text { Statistical } \\
\text { indicators } \\
\text { variables }\end{array}$} & \multicolumn{2}{|c|}{$\begin{array}{l}\text { Highest } \\
\text { Quartile }\end{array}$} & \multicolumn{2}{|c|}{$\begin{array}{l}\text { Lowest } \\
\text { Quartile }\end{array}$} & \multirow[t]{2}{*}{$\begin{array}{l}\text { Mean } \\
\text { difference }\end{array}$} & \multirow[t]{2}{*}{$\mathrm{T}$} & \multirow[t]{2}{*}{$\begin{array}{l}\text { Validity } \\
\text { coefficient }\end{array}$} \\
\hline & Mean & $\pm S D$ & Mean & $\pm S D$ & & & \\
\hline $\begin{array}{l}\text { Attention } \\
\text { Deficit }\end{array}$ & 54.53 & 2.23 & 51.73 & 3.93 & 2.8 & $\begin{array}{l}6.956 \\
* \\
\end{array}$ & 0.829 \\
\hline Hyperactivity & 53.86 & 2.85 & 50.11 & 3.58 & 3.75 & $\begin{array}{l}7.565 \\
* \quad \\
\end{array}$ & 0.850 \\
\hline Impulsivity & 49.12 & 2.25 & 44.25 & 2.03 & 4.87 & $\begin{array}{l}10.586 \\
*\end{array}$ & 0.914 \\
\hline Total & 157.51 & 5.12 & 146.09 & 6.43 & 11.42 & $\begin{array}{l}7.364 \\
* \\
\end{array}$ & 0.843 \\
\hline
\end{tabular}

* Significant at the 0.05 level $=2.23$

Table (2) results reveal significant differences between

highest and lowest quartiles as T value ranges between $(6.956$

and 10.568) values which is greater than T significant at 0.05 level.

Validity coefficient ranged between $(0.829$ and 0.914$)$, the matter that proof validity of ADHD checklist

II-Reliability:

Correlation (Reliability) coefficient calculated using test/retest method on sample of 10 the sample scoping study and which are based on (12) pupils with 7 days interval between tests. 
Table (3) Significance of differences between first and second tests of locomotor and physical tests $(n=12)$

\begin{tabular}{|c|c|c|c|c|c|c|c|c|}
\hline \multirow{2}{*}{\multicolumn{3}{|c|}{$\begin{array}{l}\text { Statistical indicators } \\
\text { physical variables }\end{array}$}} & \multicolumn{2}{|c|}{$1^{\text {st }}$ test } & \multicolumn{2}{|c|}{$2^{\text {nd }}$ test } & \multirow{2}{*}{$\begin{array}{l}\text { Mean } \\
\text { differe } \\
\text { nce }\end{array}$} & \multirow[b]{2}{*}{$\begin{array}{l}\text { Correla } \\
\text { tion } \\
\text { (Reliabi } \\
\text { lity) } \\
\text { coeffici } \\
\text { ent }\end{array}$} \\
\hline & & & $\begin{array}{l}\mathrm{Me} \\
\text { an }\end{array}$ & $\begin{array}{l} \pm S \\
D\end{array}$ & $\begin{array}{l}\text { Me } \\
\text { an }\end{array}$ & $\begin{array}{l} \pm S \\
D\end{array}$ & & \\
\hline \multicolumn{2}{|l|}{ Flexibility } & $\begin{array}{l}\text { Trunk } \\
\text { front } \\
\text { flexion } \\
\text { from } \\
\text { long } \\
\text { sitting } \\
\text { test } \\
(\mathrm{cm})\end{array}$ & $\begin{array}{l}- \\
7.3 \\
5\end{array}$ & $\begin{array}{l}3 . \\
15\end{array}$ & $\begin{array}{l}- \\
7.2 \\
3\end{array}$ & $\begin{array}{l}2 . \\
98\end{array}$ & -0.12 & 0.909 \\
\hline \multicolumn{2}{|l|}{ speed } & $\begin{array}{l}20 \mathrm{~m} \\
\text { running } \\
\text { (secon } \\
\text { d) }\end{array}$ & $\begin{array}{l}5.6 \\
5\end{array}$ & $\begin{array}{l}1 . \\
04\end{array}$ & $\begin{array}{l}5.3 \\
7\end{array}$ & $\begin{array}{l}0 . \\
96\end{array}$ & 0.28 & 0.842 \\
\hline \multicolumn{2}{|l|}{ agility } & $\begin{array}{l}\text { Zigzag } \\
\text { running } \\
\text { (secon } \\
\text { ds) }\end{array}$ & $\begin{array}{l}17 . \\
14\end{array}$ & $\begin{array}{l}1 . \\
65\end{array}$ & $\begin{array}{l}17 . \\
25\end{array}$ & $\begin{array}{l}1 . \\
84\end{array}$ & -0.11 & 0.912 \\
\hline \multirow[t]{2}{*}{$\begin{array}{l}\text { coordin } \\
\text { ation }\end{array}$} & $\begin{array}{l}\text { Eye- } \\
\text { hand } \\
\text { coordin } \\
\text { ation }\end{array}$ & $\begin{array}{l}\text { Ball } \\
\text { throw } \\
\text { and } \\
\text { receive } \\
\text { within } \\
30 \\
\text { second } \\
\text { s } \\
\text { (numbe } \\
\text { r) }\end{array}$ & $\begin{array}{l}3.1 \\
1\end{array}$ & $\begin{array}{l}1 . \\
02\end{array}$ & $\begin{array}{l}3.4 \\
7\end{array}$ & $\begin{array}{l}1 . \\
95\end{array}$ & -0.46 & 0.856 \\
\hline & $\begin{array}{l}\text { Eye- } \\
\text { legs } \\
\text { coordin } \\
\text { ation }\end{array}$ & $\begin{array}{l}\text { Numbe } \\
\text { red } \\
\text { circles } \\
\text { test } \\
\text { (secon } \\
\text { ds) }\end{array}$ & $\begin{array}{l}8.1 \\
5\end{array}$ & $\begin{array}{l}0 . \\
88\end{array}$ & $\begin{array}{l}7.9 \\
8\end{array}$ & $\begin{array}{l}0 . \\
76\end{array}$ & 0.17 & 0.915 \\
\hline Balance & $\begin{array}{l}\text { Stability } \\
\text { balance }\end{array}$ & $\begin{array}{l}\text { Standin } \\
\mathrm{g} \text { with } \\
\text { feet on }\end{array}$ & $\begin{array}{l}23 . \\
19\end{array}$ & $\begin{array}{l}1 . \\
98\end{array}$ & $\begin{array}{l}22 . \\
56\end{array}$ & $\begin{array}{l}1 . \\
87\end{array}$ & 0.63 & 0.924 \\
\hline
\end{tabular}




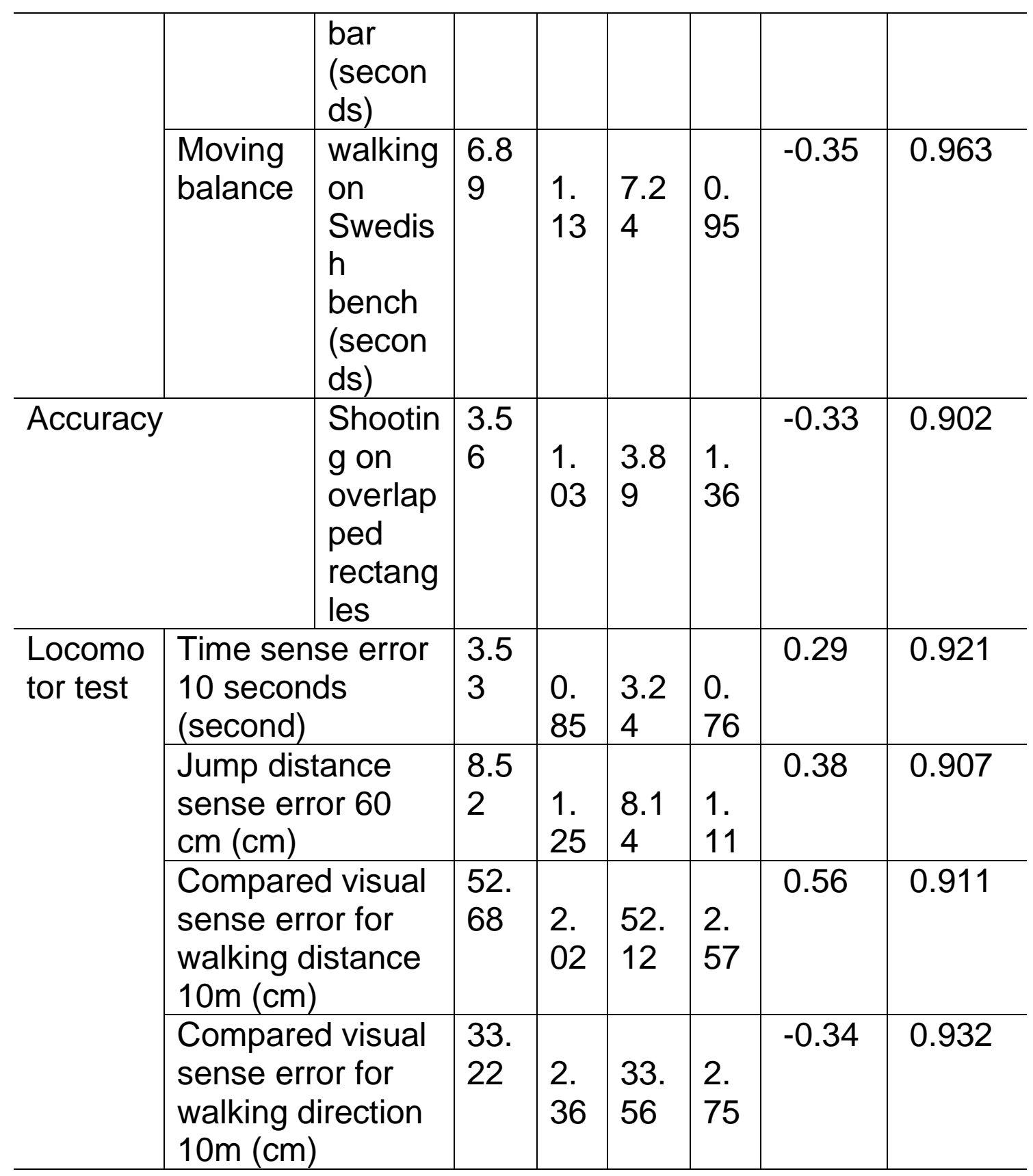

* Significant at the 0.05 level $=0.576$

Table (3) results reveal existence of statistically significant correlation between First and second application. $R$ values ranged between (0.842 and 0.963), values which are higher than $R$ significant at 0.05 .

Table (4) Significance of differences between first and second administration of ADHD checklist for learnable mentally disabled children $(n=12)$

\begin{tabular}{c|c|c|c|c|l|l}
\hline $\begin{array}{r}\text { Statistical } \\
\text { indicators } \\
\text { variables }\end{array}$ & \multicolumn{2}{|c|}{$1^{\text {st }}$ test } & \multicolumn{2}{|c|}{$2^{\text {nd }}$ test } & $\begin{array}{l}\text { Mean } \\
\text { differenc }\end{array}$ & $\begin{array}{l}\text { Correlation } \\
\text { (Reliability } \\
\text { e }\end{array}$ \\
\cline { 2 - 7 } & Mean & $\pm S D$ & Mean & $\pm S D$ & $\begin{array}{l}\text { Coefficient } \\
\text { colion }\end{array}$ \\
\hline Attention & 53.02 & & 52.92 & & 0.1 & 0.995 \\
\hline
\end{tabular}




\begin{tabular}{l|l|l|l|l|l|l}
\hline Deficit & & $\begin{array}{l}2.7 \\
2\end{array}$ & & $\begin{array}{l}3.9 \\
3\end{array}$ & & \\
\hline $\begin{array}{l}\text { Hyperactivit } \\
\mathrm{y}\end{array}$ & 52.64 & $\begin{array}{l}3.2 \\
4\end{array}$ & 52.25 & $\begin{array}{l}3.5 \\
8\end{array}$ & 0.39 & 0.925 \\
\hline Impulsivity & 47.25 & $\begin{array}{l}1.6 \\
5\end{array}$ & 46.69 & $\begin{array}{l}1.8 \\
4\end{array}$ & 0.56 & 0.911 \\
\hline Total & 152.9 & $\begin{array}{l}6.7 \\
3\end{array}$ & $\begin{array}{l}151.8 \\
6\end{array}$ & $\begin{array}{l}7.6 \\
8\end{array}$ & 1.05 & 0.945 \\
\hline
\end{tabular}

* Significant at the 0.05 level $=0.576$

Table (4) results reveal existence of statistically significant correlation between First and second application. $R$ values ranged between (0.911 and 0.995), values which are higher than $R$ significant at 0.05 .

Statistical Analysis:

SPSS statistical program used to extract the following:

Mean- standard deviation - skewness coefficient - Means difference $-T$ - validity coefficient - reliability coefficient - variance coefficient.

Sample homogeneity

Table (5) overall research basic variables descriptive statistics sample before experiment $\mathbf{n}=\mathbf{2 4}$

\begin{tabular}{l|l|l|l|l}
\hline $\begin{array}{c}\text { Statistical indicators } \\
\text { Basic variables }\end{array}$ & Mean & $\pm S D$ & $\begin{array}{l}\text { Skewness } \\
\text { coefficient }\end{array}$ & $\begin{array}{l}\text { Variance } \\
\text { coefficient }\end{array}$ \\
\hline Height $(\mathrm{cm})$ & 136.90 & 6.24 & 0.62 & 4.558 \\
\hline Weight $(\mathrm{kg})$ & 35.86 & 3.95 & 0.35 & 11.015 \\
\hline Age & 11.35 & 1.14 & 0.53 & 10.044 \\
\hline Mental age & 8.12 & 1.02 & 0.12 & 12.562 \\
\hline IQ & 59.34 & 4.25 & 0.98 & 7.162 \\
\hline
\end{tabular}

Table (5) results reveal skewness coefficients values between ( 0.12 and 0.98$)$, values which is between \pm 3 and close to zero; the matter which confirms that sample is free of abnormal distributions defects. Results also reveal that variance coefficient for all basic variables for overall sample is less than $20 \%$, which demonstrates homogeneity of sample individuals in basic variables before experiment.

Table (6) Significance of differences between experimental and control groups in basic variables before experiment \begin{tabular}{l|l|l|l|l} 
Statistical Experimental & Control & Mean & T
\end{tabular} 


\begin{tabular}{|c|c|c|c|c|c|c|}
\hline indicators & group ( & $\mathrm{n=12)}$ & $\begin{array}{l}\text { grol } \\
(n=1\end{array}$ & & difference & \\
\hline Basic variables & Mean & $\pm S D$ & Mean & $\pm S D$ & & \\
\hline Height $(\mathrm{cm})$ & 137.56 & 7.12 & 136.32 & 6.46 & 1.24 & 0.447 \\
\hline Weight (kg) & 36.23 & 4.13 & 35.37 & 3.78 & 0.86 & 0.532 \\
\hline Age & 11.02 & 1.19 & 11.64 & 1.05 & -0.62 & 1.354 \\
\hline Mental age & 8.02 & 1.15 & 8.35 & 1.36 & -0.33 & 0.642 \\
\hline $\mathrm{IQ}$ & 59.11 & 5.72 & 59.54 & 4.98 & -0.43 & 0.196 \\
\hline
\end{tabular}

* Significant at the 0.05 level $=2.074$

Table (6) results reveal that differences between experimental and control groups in basic variables are not significant as $T$ value ranges between $(0.196$ and 1.534$)$ values which is less than $T$ significant at 0.05 level, the matter that proof research group homogeneity in these variables

\section{Table (7) Significance of differences between experimental} and control groups in locomotor and physical tests before experiment

\begin{tabular}{|c|c|c|c|c|c|c|c|}
\hline \multirow{2}{*}{\multicolumn{3}{|c|}{ physical variables }} & \multicolumn{2}{|c|}{$\begin{array}{l}\text { Experimental } \\
\text { group }(\mathbf{n}=\mathbf{1 2})\end{array}$} & \multicolumn{2}{|c|}{$\begin{array}{c}\text { Control } \\
\text { group } \\
(\mathbf{n}=12)\end{array}$} & \multirow[t]{2}{*}{$\begin{array}{l}\text { Mean } \\
\text { difference }\end{array}$} \\
\hline & & & Mean & $\pm S D$ & Mean & $\pm S D$ & \\
\hline Flexibility & & $\begin{array}{l}\text { Trunk front } \\
\text { flexion } \\
\text { from long } \\
\text { sitting test } \\
\text { (cm) }\end{array}$ & -7.18 & 3.52 & -7.42 & 3.74 & 0.24 \\
\hline speed & & $\begin{array}{l}20 \mathrm{~m} \\
\text { running } \\
\text { (second) }\end{array}$ & 5.31 & 0.64 & 5.02 & 0.82 & 0.29 \\
\hline agility & & $\begin{array}{l}\text { Zigzag } \\
\text { running } \\
\text { (seconds) }\end{array}$ & 17.35 & 1.84 & 17.92 & 2.13 & -0.57 \\
\hline coordination & $\begin{array}{l}\text { Eye-hand } \\
\text { coordination }\end{array}$ & $\begin{array}{l}\text { Ball throw } \\
\text { and } \\
\text { receive } \\
\text { within } 30 \\
\text { seconds }\end{array}$ & 3.25 & 1.73 & 3.61 & 1.41 & -0.36 \\
\hline
\end{tabular}




\begin{tabular}{|c|c|c|c|c|c|c|c|}
\hline & & (number) & & & & & \\
\hline & $\begin{array}{l}\text { Eye-legs } \\
\text { coordination }\end{array}$ & $\begin{array}{l}\text { Numbered } \\
\text { circles test } \\
\text { (seconds) }\end{array}$ & 8.35 & 0.74 & 8.49 & 0.98 & -0.14 \\
\hline \multirow[t]{2}{*}{ Balance } & $\begin{array}{l}\text { Stability } \\
\text { balance }\end{array}$ & $\begin{array}{l}\text { Standing } \\
\text { with feet } \\
\text { on bar } \\
\text { (seconds) }\end{array}$ & 22.63 & 2.02 & 21.98 & 1.95 & 0.65 \\
\hline & $\begin{array}{l}\text { Moving } \\
\text { balance }\end{array}$ & $\begin{array}{l}\text { walking on } \\
\text { Swedish } \\
\text { bench } \\
\text { (seconds) }\end{array}$ & 7.35 & 0.84 & 6.97 & 1.03 & 0.38 \\
\hline \multicolumn{2}{|l|}{ Accuracy } & $\begin{array}{l}\text { Shooting } \\
\text { on } \\
\text { overlapped } \\
\text { rectangles }\end{array}$ & 3.29 & 0.97 & 3.43 & 1.21 & -0.14 \\
\hline \multirow[t]{4}{*}{$\begin{array}{l}\text { Locomotor } \\
\text { test }\end{array}$} & \multicolumn{2}{|c|}{$\begin{array}{l}\text { Time sense error } 10 \\
\text { seconds (second) }\end{array}$} & 3.21 & 0.26 & 3.11 & 0.32 & 0.1 \\
\hline & \multicolumn{2}{|c|}{$\begin{array}{l}\text { Jump distance sense } \\
\text { error } 60 \mathrm{~cm}(\mathrm{~cm})\end{array}$} & 8.78 & 0.96 & 8.35 & 0.89 & 0.43 \\
\hline & \multicolumn{2}{|c|}{$\begin{array}{l}\text { Compared visual sense } \\
\text { error for walking } \\
\text { distance } 10 \mathrm{~m}(\mathrm{~cm})\end{array}$} & 53.56 & 1.93 & 52.26 & 2.23 & 2.3 \\
\hline & \multicolumn{2}{|c|}{$\begin{array}{l}\text { Compared visual sense } \\
\text { error for walking } \\
\text { direction } 10 \mathrm{~m}(\mathrm{~cm})\end{array}$} & 33.54 & 1.95 & 34.26 & 2.36 & -0.72 \\
\hline
\end{tabular}

* Significant at the 0.05 level $=2.074$

Table (7) results reveal that differences between experimental and control groups in locomotor and physical tests are not significant as $T$ value ranges between ( 0.157 and 1.523$)$ values which is less than $T$ significant at 0.05 level, the matter that proof research group homogeneity in these variables

Table (8) Significance of differences between experimental and control groups in ADHD checklist for learnable mentally disabled children

\begin{tabular}{|c|c|c|c|c|c|c|}
\hline \multirow{2}{*}{$\begin{array}{l}\text { Statistical } \\
\text { indicators } \\
\text { variables }\end{array}$} & \multicolumn{2}{|c|}{$\begin{array}{l}\text { Experimental } \\
\text { group }(\mathrm{n}=12)\end{array}$} & \multicolumn{2}{|c|}{$\begin{array}{l}\text { Control group } \\
(\mathbf{n}=12)\end{array}$} & \multirow[t]{2}{*}{$\begin{array}{l}\text { Mean } \\
\text { difference }\end{array}$} & \multirow[t]{2}{*}{$\mathrm{T}$} \\
\hline & Mean & $\pm S D$ & Mean & $\pm S D$ & & \\
\hline $\begin{array}{l}\text { Attention } \\
\text { Deficit }\end{array}$ & 53.65 & 3.18 & 53.16 & 3.57 & 0.49 & 0.257 \\
\hline Hyperactivity & 52.68 & 2.96 & 52.87 & 2.67 & -0.19 & 0.165 \\
\hline
\end{tabular}




\begin{tabular}{|c|c|c|c|c|c|c|}
\hline Impulsivity & 48.39 & 2.24 & 47.98 & 1.93 & 0.41 & 0.480 \\
\hline Total & 154.72 & 7.15 & 154.01 & 7.37 & 0.71 & 0.240 \\
\hline
\end{tabular}

${ }^{*}$ Significant at the 0.05 level $=2.074$

Table (8) results reveal that differences between experimental and control groups in ADHD checklist are not significant as $T$ value ranges between $(0.165$ and 0.480$)$ values which is less than $T$ significant at 0.05 level, the matter that proof research group homogeneity in these variables.

\section{Main study:}

Coordination exercises training aiming at improve some locomotor and physical abilities and ease ADHD designed to be applied in ten (10) consecutive weeks, three (3) training units per week, each unit is 45 minutes, the study started by implementing the program as follows:

\section{Experimental group:}

Undergone training program consists of (30) training unit applied on (Saturday - Monday - Wednesday) each week.

\section{Control group:}

Undergone school motor syllabus in physical education classes on (Saturday - Monday - Wednesday) each week.

\section{The proposed program:}

\section{Training unit components:}

- Warm-up: duration (7) minutes, contains exercises in form of games aiming to muscles general preparation, stimulate blood circulation and prepare body joints for effort.

- Main part: duration (35) minutes, includes physical exercise.

- Cool down: duration (3) minutes, contains exercises to calm body and return to nearly normal state.

\section{Program development foundations:}

- Using low intensity interval-training method for developing physical abilities.

- Adopting load/rest principle as a training base.

- Load gradual rise.

- Right time for exercise repetition.

- Continuity in performing exercise and training. (Allawi, 1994, p12; Ahmed, 1999, p .288; and Abbas, 2005, p. 178-179)

- Taken into account when developing and implementing program exercises to be in form of gradual difficulty training unit.

- Each training unit included (3) warm-up exercises (for functional preparation) performed individually. 
- Each training unit included (5) physical preparation exercises (to develop physical abilities).

- Each training unit included (2) cool down exercises

- (50: 70\%) intensity from pupil maximum level while performing physical exercises. (Figure 1)

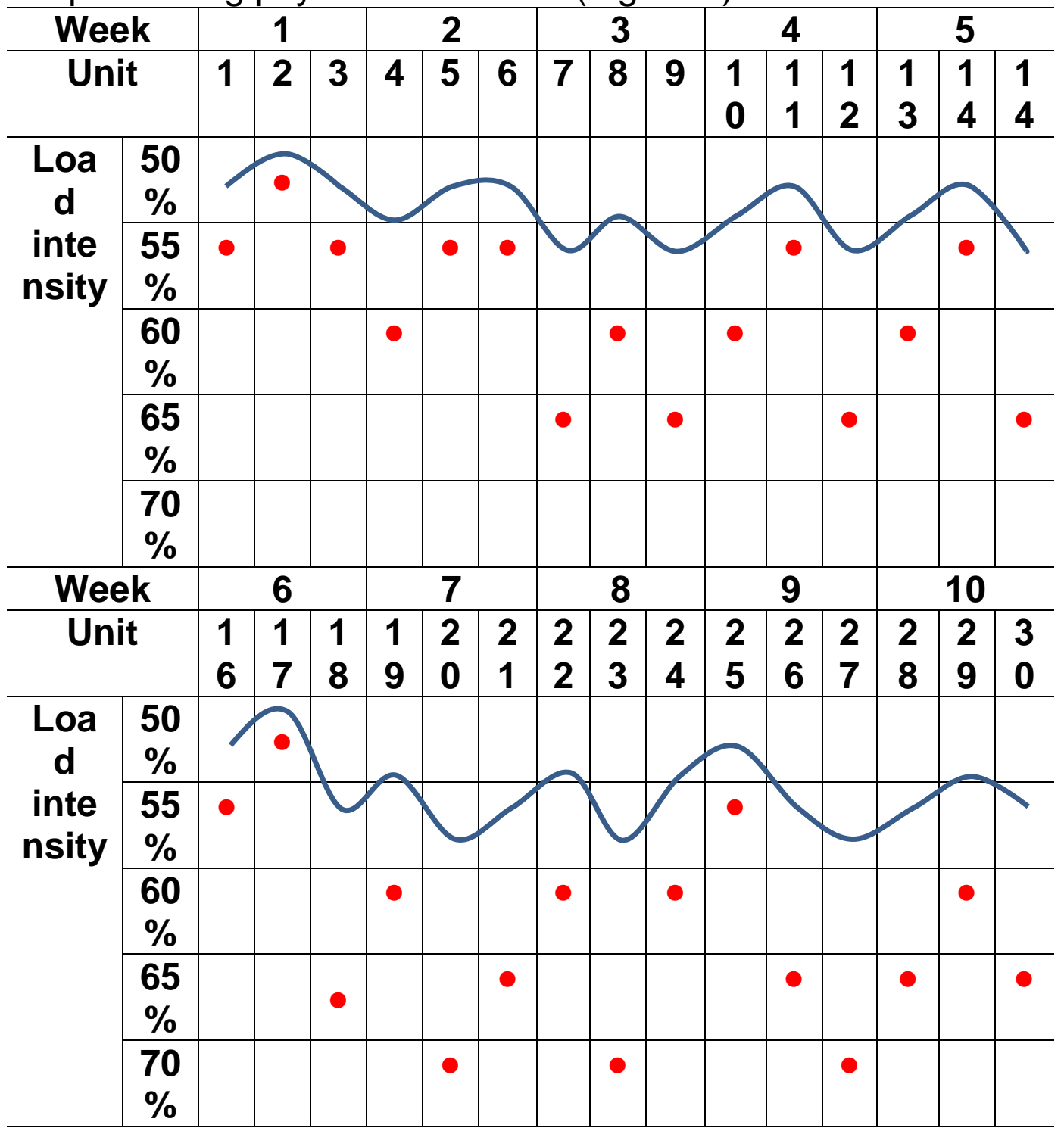

Figure (1) Load intensity distribution on weekly units

- The program contains exercises without tools, exercises using body as weight, exercises with tools (balls - seats hoops- Sand bags - Wands).

\section{Rationing exercise in terms of time:}

- $50 \%$ of pupil maximum repetition calculated exercise performance. (Maximum repetition $\times 50 / 100$ ).

- Maximum time for each exercise performance calculated for each exercise separately.

- Performance time for each exercise is (30) seconds, with (40: 45) seconds interval rest. 
- The number of groups is (4) groups, and interval rest between groups is (2: 3$)$ minutes.

- Results

Table (9) Significance of differences between experimental group pre and post measurements of locomotor and physical tests $(n=12)$

\begin{tabular}{|c|c|c|c|c|c|c|c|}
\hline \multirow{2}{*}{\multicolumn{3}{|c|}{$\begin{array}{l}\text { Statistical indicators } \\
\text { physical variables }\end{array}$}} & \multicolumn{2}{|c|}{$\begin{array}{c}\text { Pre } \\
\text { measurement }\end{array}$} & \multicolumn{2}{|c|}{$\begin{array}{c}\text { Post } \\
\text { measurement }\end{array}$} & \multirow[t]{3}{*}{$\begin{array}{l}\text { Mean } \\
\text { difference }\end{array}$} \\
\hline & & & Mean & $\pm S D$ & Mean & $\pm S D$ & \\
\hline \multicolumn{2}{|l|}{ Flexibility } & $\begin{array}{l}\text { Trunk front } \\
\text { flexion } \\
\text { from long } \\
\text { sitting test } \\
\text { (cm) }\end{array}$ & -7.18 & 3.52 & -1.12 & 2.43 & \\
\hline \multicolumn{2}{|l|}{ speed } & $\begin{array}{l}20 \mathrm{~m} \\
\text { running } \\
\text { (second) }\end{array}$ & 5.31 & 0.64 & 4.12 & 0.79 & 1.19 \\
\hline \multicolumn{2}{|l|}{ agility } & $\begin{array}{l}\text { Zigzag } \\
\text { running } \\
\text { (seconds) }\end{array}$ & 17.35 & 1.84 & 12.29 & 0.94 & 5.06 \\
\hline \multirow[t]{2}{*}{ coordination } & $\begin{array}{l}\text { Eye-hand } \\
\text { coordination }\end{array}$ & $\begin{array}{l}\text { Ball throw } \\
\text { and } \\
\text { receive } \\
\text { within } 30 \\
\text { seconds } \\
\text { (number) }\end{array}$ & 3.25 & 1.73 & 8.56 & 1.52 & -5.31 \\
\hline & $\begin{array}{l}\text { Eye-legs } \\
\text { coordination }\end{array}$ & $\begin{array}{l}\text { Numbered } \\
\text { circles test } \\
\text { (seconds) }\end{array}$ & 8.35 & 0.74 & 5.92 & 0.65 & 2.43 \\
\hline \multirow[t]{2}{*}{ Balance } & $\begin{array}{l}\text { Stability } \\
\text { balance }\end{array}$ & $\begin{array}{l}\text { Standing } \\
\text { with feet } \\
\text { on bar } \\
\text { (seconds) }\end{array}$ & 22.63 & 2.02 & 30.56 & 2.15 & 7.93 \\
\hline & $\begin{array}{l}\text { Moving } \\
\text { balance }\end{array}$ & $\begin{array}{l}\text { walking on } \\
\text { Swedish } \\
\text { bench } \\
\text { (seconds) }\end{array}$ & 7.35 & 0.84 & 5.49 & 0.62 & 1.86 \\
\hline \multicolumn{2}{|l|}{ Accuracy } & $\begin{array}{l}\text { Shooting } \\
\text { on } \\
\text { overlapped } \\
\text { rectangles }\end{array}$ & 3.29 & 0.97 & 6.14 & 1.93 & -2.85 \\
\hline Locomotor & \multicolumn{2}{|c|}{ Time sense error 10} & 3.21 & 0.26 & 1.71 & 0.72 & 1.5 \\
\hline
\end{tabular}




\begin{tabular}{|c|c|c|c|c|c|c|}
\hline \multirow{4}{*}{ test } & seconds (second) & & & & & \\
\hline & $\begin{array}{l}\text { Jump distance sense } \\
\text { error } 60 \mathrm{~cm}(\mathrm{~cm})\end{array}$ & 8.78 & 0.96 & 3.36 & 0.95 & 5.42 \\
\hline & $\begin{array}{l}\text { Compared visual sense } \\
\text { error for walking } \\
\text { distance } 10 \mathrm{~m}(\mathrm{~cm})\end{array}$ & 53.56 & 1.93 & 37.68 & 1.58 & 15.88 \\
\hline & $\begin{array}{l}\text { Compared visual sense } \\
\text { error for walking } \\
\text { direction } 10 \mathrm{~m}(\mathrm{~cm})\end{array}$ & 33.54 & 1.95 & 23.23 & 2.31 & 10.31 \\
\hline
\end{tabular}

* Significant at the 0.05 level $=2.201$

Table (9) results reveal significant differences between experimental group pre and post measurements in locomotor and physical tests as T value ranges between (6.06 and 24.06) values which is greater than $T$ significant at 0.05 level, with improvement percentage range between (22.41 and 163.38) in favor of post measurement in all variables .

Table (10) Significance of differences between experimental group pre and post measurements of ADHD checklist $(n=12)$

\begin{tabular}{|c|c|c|c|c|c|c|c|}
\hline \multirow{2}{*}{$\begin{array}{l}\text { Statistical } \\
\text { indicators } \\
\text { physical } \\
\text { variables }\end{array}$} & \multicolumn{2}{|c|}{$\begin{array}{c}\text { Pre } \\
\text { measurem } \\
\text { ent }\end{array}$} & \multicolumn{2}{|c|}{$\begin{array}{c}\text { Post } \\
\text { measurem } \\
\text { ent }\end{array}$} & \multirow[t]{2}{*}{$\begin{array}{l}\text { Mean } \\
\text { differen } \\
\text { ce }\end{array}$} & \multirow[t]{2}{*}{$\mathrm{T}$} & \multirow[t]{2}{*}{$\begin{array}{l}\text { Improvem } \\
\text { ent } \\
\%\end{array}$} \\
\hline & $\begin{array}{l}\text { Mea } \\
n\end{array}$ & $\pm S D$ & $\begin{array}{l}\text { Mea } \\
n\end{array}$ & $\pm S D$ & & & \\
\hline $\begin{array}{l}\text { Attention } \\
\text { Deficit }\end{array}$ & $\begin{array}{l}53.6 \\
5\end{array}$ & 3.18 & $\begin{array}{l}37.1 \\
9\end{array}$ & 3.76 & 16.46 & $\begin{array}{l}11.9 \\
7^{*}\end{array}$ & 30.68 \\
\hline $\begin{array}{l}\text { Hyperacti } \\
\text { vity }\end{array}$ & $\begin{array}{l}52.6 \\
8\end{array}$ & 2.96 & $\begin{array}{l}35.6 \\
7\end{array}$ & 3.35 & 17.01 & $\begin{array}{l}15.1 \\
3^{*}\end{array}$ & 32.29 \\
\hline $\begin{array}{l}\text { Impulsivit } \\
y\end{array}$ & $\begin{array}{l}48.3 \\
9\end{array}$ & 2.24 & $\begin{array}{l}29.3 \\
6\end{array}$ & 2.95 & 19.03 & $\begin{array}{l}21.2 \\
4^{*}\end{array}$ & 39.33 \\
\hline Total & $\begin{array}{l}154 . \\
72\end{array}$ & 7.15 & $\begin{array}{l}102 . \\
22\end{array}$ & 7.68 & 52.5 & $\begin{array}{l}26.5 \\
6^{*}\end{array}$ & 33.93 \\
\hline
\end{tabular}

${ }^{*}$ Significant at the 0.05 level $=2.201$

Table (10) results reveal significant differences between experimental group pre and post measurements in ADHD checklist as $\mathrm{T}$ value ranges between (11.97 and 26.56) values which is 
greater than $\mathrm{T}$ significant at 0.05 level, with improvement percentage range between (30.68 and 39.33) in favor of post measurement in all variables .

Table (11) Significance of differences between control group pre and post measurements of locomotor and physical tests

\begin{tabular}{|c|c|c|c|c|c|c|c|c|c|}
\hline \multirow{3}{*}{\multicolumn{3}{|c|}{ phys }} & \multicolumn{4}{|c|}{$(n=12)$} & \multirow{3}{*}{$\begin{array}{l}\text { Mean } \\
\text { differ } \\
\text { ence }\end{array}$} & \multirow{3}{*}{$\mathrm{T}$} & \multirow{3}{*}{$\begin{array}{l}\text { Improv } \\
\text { ement } \\
\%\end{array}$} \\
\hline & & & \multicolumn{2}{|c|}{$\begin{array}{c}\text { Pre } \\
\text { measur } \\
\text { ement }\end{array}$} & \multicolumn{2}{|c|}{$\begin{array}{c}\text { Post } \\
\text { measur } \\
\text { ement }\end{array}$} & & & \\
\hline & & & $\begin{array}{l}\text { Me } \\
\text { an }\end{array}$ & \begin{tabular}{|l} 
$\pm S$ \\
$D$ \\
\end{tabular} & $\begin{array}{l}\text { Me } \\
\text { an }\end{array}$ & $\begin{array}{l} \pm S \\
D\end{array}$ & & & \\
\hline Flexibili & & $\begin{array}{l}\text { Trunk } \\
\text { front } \\
\text { flexio } \\
\text { n from } \\
\text { long } \\
\text { sitting } \\
\text { test } \\
\text { (cm) }\end{array}$ & $\begin{array}{l}- \\
7.4 \\
2\end{array}$ & $\begin{array}{l}3.7 \\
4\end{array}$ & $\begin{array}{l}- \\
5.3 \\
2\end{array}$ & $\begin{array}{l}1.9 \\
2\end{array}$ & -2.1 & $\begin{array}{l}3.7 \\
3 \text { * }\end{array}$ & 28.30 \\
\hline speed & & $\begin{array}{l}20 \mathrm{~m} \\
\text { runnin } \\
\mathrm{g} \\
\text { (seco } \\
\text { nd) }\end{array}$ & $\begin{array}{l}5.0 \\
2\end{array}$ & $\begin{array}{l}0.8 \\
2\end{array}$ & $\begin{array}{l}4.8 \\
1\end{array}$ & $\begin{array}{l}0.5 \\
9\end{array}$ & 0.21 & $\begin{array}{l}2.3 \\
7 \text { * }\end{array}$ & 4.18 \\
\hline agility & & $\begin{array}{l}\text { Zigza } \\
\text { g } \\
\text { runnin } \\
\text { g } \\
\text { (seco } \\
\text { nds) }\end{array}$ & $\begin{array}{l}17 . \\
92\end{array}$ & $\begin{array}{l}2.1 \\
3\end{array}$ & $\begin{array}{l}16 . \\
93\end{array}$ & $\begin{array}{l}0.8 \\
5\end{array}$ & 0.99 & $\begin{array}{l}3.5 \\
*\end{array}$ & 5.52 \\
\hline $\begin{array}{l}\text { coordi } \\
\text { nation }\end{array}$ & $\begin{array}{l}\text { Eye- } \\
\text { hand } \\
\text { coordi } \\
\text { nation }\end{array}$ & $\begin{array}{l}\text { Ball } \\
\text { throw } \\
\text { and } \\
\text { receiv } \\
\text { e } \\
\text { within } \\
30 \\
\text { secon } \\
\text { ds } \\
\text { (numb } \\
\text { er) }\end{array}$ & $\begin{array}{l}3.6 \\
1\end{array}$ & $\begin{array}{l}1.4 \\
1\end{array}$ & $\begin{array}{l}4.4 \\
2\end{array}$ & $\begin{array}{l}1.4 \\
9\end{array}$ & 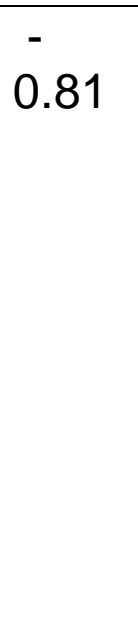 & $\begin{array}{l}3.3 \\
7 \text { * }\end{array}$ & 22.44 \\
\hline & $\begin{array}{l}\text { Eye- } \\
\text { legs }\end{array}$ & $\begin{array}{l}\text { Numb } \\
\text { ered }\end{array}$ & $\begin{array}{l}8.4 \\
9\end{array}$ & 0.9 & 7.9 & $\begin{array}{l}0.6 \\
4\end{array}$ & 0.58 & $\begin{array}{l}3.7 \\
2 \text { * }\end{array}$ & 6.83 \\
\hline
\end{tabular}




\begin{tabular}{|c|c|c|c|c|c|c|c|c|c|}
\hline & $\begin{array}{l}\text { coordi } \\
\text { nation }\end{array}$ & $\begin{array}{l}\text { circles } \\
\text { test } \\
\text { (seco } \\
\text { nds) } \\
\end{array}$ & & 8 & 1 & & & & \\
\hline \multirow[t]{2}{*}{$\begin{array}{l}\text { Balanc } \\
\mathrm{e}\end{array}$} & $\begin{array}{l}\text { Stabilit } \\
\text { y } \\
\text { balanc } \\
\text { e }\end{array}$ & \begin{tabular}{|l} 
Standi \\
ng \\
with \\
feet \\
on bar \\
(seco \\
nds) \\
\end{tabular} & $\begin{array}{l}21 . \\
98\end{array}$ & $\begin{array}{l}1.9 \\
5\end{array}$ & 23. & $\begin{array}{l}2.6 \\
4\end{array}$ & $-\overline{1.55}$ & $\begin{array}{l}3.6 \\
3 \text { * }\end{array}$ & 7.05 \\
\hline & $\begin{array}{l}\text { Movin } \\
g \\
\text { balanc } \\
\text { e }\end{array}$ & \begin{tabular}{|l} 
walkin \\
g on \\
Swedi \\
sh \\
bench \\
(seco \\
nds) \\
\end{tabular} & $\begin{array}{l}6.9 \\
7\end{array}$ & $\begin{array}{l}1.0 \\
3\end{array}$ & $\begin{array}{l}6.7 \\
4\end{array}$ & $\begin{array}{l}0.8 \\
5\end{array}$ & 0.23 & $\begin{array}{l}2.5 \\
9 \text { * }\end{array}$ & 3.29 \\
\hline \multicolumn{2}{|c|}{ Accuracy } & $\begin{array}{l}\text { Shooti } \\
\text { ng on } \\
\text { overla } \\
\text { pped } \\
\text { rectan } \\
\text { gles }\end{array}$ & $\begin{array}{l}3.4 \\
3\end{array}$ & $\begin{array}{l}1.2 \\
1\end{array}$ & $\begin{array}{l}4.0 \\
2\end{array}$ & $\begin{array}{l}2.1 \\
4\end{array}$ & $-\overline{0.59}$ & $\begin{array}{l}2.8 \\
3 \text { * }\end{array}$ & 17.20 \\
\hline \multirow[t]{4}{*}{$\begin{array}{l}\text { Locom } \\
\text { otor } \\
\text { test }\end{array}$} & \multicolumn{2}{|c|}{$\begin{array}{l}\text { Time sense } \\
\text { error } 10 \\
\text { seconds } \\
\text { (second) }\end{array}$} & $\begin{array}{l}3.1 \\
1\end{array}$ & $\begin{array}{l}0.3 \\
2\end{array}$ & $\begin{array}{l}2.7 \\
5\end{array}$ & $\begin{array}{l}1.2 \\
3\end{array}$ & 0.36 & $\begin{array}{l}2.9 \\
8 \text { * }\end{array}$ & 11.58 \\
\hline & \multicolumn{2}{|c|}{$\begin{array}{l}\text { Jump distance } \\
\text { sense error } 60 \\
\mathrm{~cm}(\mathrm{~cm})\end{array}$} & $\begin{array}{l}8.3 \\
5\end{array}$ & $\begin{array}{l}0.8 \\
9\end{array}$ & $\begin{array}{l}6.9 \\
3\end{array}$ & $\begin{array}{l}1.7 \\
8\end{array}$ & 1.42 & $\begin{array}{l}4.4 \\
9 \text { * }\end{array}$ & 17.01 \\
\hline & \multicolumn{2}{|c|}{$\begin{array}{l}\text { Compared } \\
\text { visual sense } \\
\text { error for } \\
\text { walking } \\
\text { distance } 10 \mathrm{~m} \\
\text { (cm) }\end{array}$} & $\begin{array}{l}52 . \\
26\end{array}$ & $\begin{array}{l}2.2 \\
3\end{array}$ & $\begin{array}{l}43 . \\
23\end{array}$ & $\begin{array}{l}2.1 \\
7\end{array}$ & 9.03 & $\begin{array}{l}11 . \\
85^{*}\end{array}$ & 17.28 \\
\hline & \multicolumn{2}{|c|}{$\begin{array}{l}\text { Compared } \\
\text { visual sense } \\
\text { error for } \\
\text { walking } \\
\text { direction } 10 \mathrm{~m} \\
\text { (cm) }\end{array}$} & $\begin{array}{l}34 . \\
26\end{array}$ & $\begin{array}{l}2.3 \\
6\end{array}$ & $\begin{array}{l}31 . \\
73\end{array}$ & $\begin{array}{l}2.8 \\
6\end{array}$ & 2.53 & $\begin{array}{l}4.3 \\
6 \text { * }\end{array}$ & 7.38 \\
\hline
\end{tabular}


* Significant at the 0.05 level $=2.201$

Table (11) results reveal significant differences between control group pre and post measurements in locomotor and physical tests as $T$ value ranges between (2.37 and 11.85$)$ values which is greater than $T$ significant at 0.05 level, with improvement percentage range between (3.29 and 28.30) in favor of post measurement in all variables .

Table (12) Significance of differences between control group pre and post measurements of ADHD checklist $(n=12)$

\begin{tabular}{|c|c|c|c|c|c|c|c|}
\hline \multirow{2}{*}{$\begin{array}{l}\text { Statistical } \\
\text { indicators } \\
\text { physical } \\
\text { variables }\end{array}$} & \multicolumn{2}{|c|}{$\begin{array}{c}\text { Pre } \\
\text { measureme } \\
\text { nt }\end{array}$} & \multicolumn{2}{|c|}{$\begin{array}{c}\text { Post } \\
\text { measureme } \\
\text { nt }\end{array}$} & \multirow[t]{2}{*}{$\begin{array}{l}\text { Mean } \\
\text { differen } \\
\text { ce }\end{array}$} & \multirow[t]{2}{*}{$\mathrm{T}$} & \multirow[t]{2}{*}{$\begin{array}{l}\text { Improvem } \\
\text { ent } \\
\%\end{array}$} \\
\hline & Mean & $\begin{array}{l} \pm S \\
D\end{array}$ & Mean & $\begin{array}{l} \pm S \\
D\end{array}$ & & & \\
\hline $\begin{array}{l}\text { Attention } \\
\text { Deficit }\end{array}$ & 53.16 & $\begin{array}{l}3.5 \\
7\end{array}$ & 46.36 & $\begin{array}{l}5.1 \\
2\end{array}$ & 6.8 & $\begin{array}{l}4.1 \\
5 \text { * }\end{array}$ & 12.79 \\
\hline $\begin{array}{l}\text { Hyperactiv } \\
\text { ity }\end{array}$ & 52.87 & $\begin{array}{l}2.6 \\
7\end{array}$ & 48.86 & $\begin{array}{l}4.7 \\
5\end{array}$ & 4.01 & $\begin{array}{l}2.6 \\
5^{*}\end{array}$ & 7.58 \\
\hline Impulsivity & 47.98 & $\begin{array}{l}1.9 \\
3\end{array}$ & 44.57 & $\begin{array}{l}4.6 \\
7\end{array}$ & 3.41 & $\begin{array}{l}2.3 \\
7 \text { * }\end{array}$ & 7.11 \\
\hline Total & $\begin{array}{l}154.0 \\
1\end{array}$ & $\begin{array}{l}7.3 \\
7 \\
\end{array}$ & $\begin{array}{l}139.7 \\
9\end{array}$ & $\begin{array}{l}8.4 \\
2 \\
\end{array}$ & 14.22 & $\begin{array}{l}3.1 \\
6 \text { * }\end{array}$ & 9.23 \\
\hline
\end{tabular}

* Significant at the 0.05 level $=2.201$

Table (12) results reveal significant differences between control group pre and post measurements in ADHD checklist as T value ranges between (2.37 and 4.15$)$ values which is greater than $\mathrm{T}$ significant at 0.05 level, with improvement percentage range between (7.11 and 12.79) in favor of post measurement in all variables .

Table (13) Significance of differences between experimental and control groups in locomotor and physical tests after experiment

\begin{tabular}{|c|c|c|c|c|c|c|c|}
\hline \multirow{2}{*}{\multicolumn{2}{|c|}{$\begin{array}{l}\text { Statistical indicators } \\
\text { physical variables }\end{array}$}} & \multicolumn{2}{|c|}{$\begin{array}{c}\text { Experime } \\
\text { ntal } \\
\text { group } \\
(\mathbf{n}=12)\end{array}$} & \multicolumn{2}{|c|}{$\begin{array}{c}\text { Control } \\
\text { group } \\
(n=12)\end{array}$} & \multirow[t]{2}{*}{$\begin{array}{l}\text { Mean } \\
\text { differe } \\
\text { nce }\end{array}$} & \multirow[t]{2}{*}{$\mathrm{T}$} \\
\hline & & $\begin{array}{l}\text { Mea } \\
\mathrm{n}\end{array}$ & $\begin{array}{l} \pm S \\
D\end{array}$ & $\begin{array}{l}\text { Me } \\
\text { an }\end{array}$ & $\begin{array}{l} \pm S \\
D\end{array}$ & & \\
\hline Flexibility & $\begin{array}{l}\text { Trunk } \\
\text { front } \\
\text { flexion } \\
\text { from } \\
\end{array}$ & $\overline{-}-1.12$ & $\begin{array}{l}2.4 \\
3\end{array}$ & $\begin{array}{l}- \\
5.3 \\
2\end{array}$ & $\begin{array}{l}1 . \\
92\end{array}$ & 4.2 & $\begin{array}{l}4.69 \\
8^{*}\end{array}$ \\
\hline
\end{tabular}




\begin{tabular}{|c|c|c|c|c|c|c|c|c|}
\hline & & $\begin{array}{l}\text { long } \\
\text { sitting } \\
\text { test } \\
(\mathrm{cm})\end{array}$ & & & & & & \\
\hline \multicolumn{2}{|l|}{ speed } & $\begin{array}{l}20 \mathrm{~m} \\
\text { running } \\
\text { (secon } \\
\text { d) }\end{array}$ & 4.12 & $\begin{array}{l}0.7 \\
9\end{array}$ & $\begin{array}{l}4.8 \\
1\end{array}$ & $\begin{array}{l}0 . \\
59\end{array}$ & 0.69 & $\begin{array}{l}2.42 \\
1^{*}\end{array}$ \\
\hline \multicolumn{2}{|l|}{ agility } & $\begin{array}{l}\text { Zigzag } \\
\text { running } \\
\text { (secon } \\
\text { ds) }\end{array}$ & $\begin{array}{l}12.2 \\
9\end{array}$ & $\begin{array}{l}0.9 \\
4\end{array}$ & $\begin{array}{l}16 . \\
93\end{array}$ & $\begin{array}{l}0 . \\
85\end{array}$ & -4.64 & $\begin{array}{l}12.6 \\
78^{*}\end{array}$ \\
\hline \multirow[t]{2}{*}{$\begin{array}{l}\text { coordina } \\
\text { tion }\end{array}$} & $\begin{array}{l}\text { Eye- } \\
\text { hand } \\
\text { coordina } \\
\text { tion }\end{array}$ & $\begin{array}{l}\text { Ball } \\
\text { throw } \\
\text { and } \\
\text { receive } \\
\text { within } \\
30 \\
\text { second } \\
\text { s } \\
\text { (numbe } \\
\text { r) }\end{array}$ & 8.56 & $\begin{array}{l}1.5 \\
2\end{array}$ & $\begin{array}{l}4.4 \\
2\end{array}$ & $\begin{array}{l}1 . \\
49\end{array}$ & 4.14 & $\begin{array}{l}6.74 \\
3^{*}\end{array}$ \\
\hline & $\begin{array}{l}\text { Eye- } \\
\text { legs } \\
\text { coordina } \\
\text { tion }\end{array}$ & $\begin{array}{l}\text { Numbe } \\
\text { red } \\
\text { circles } \\
\text { test } \\
\text { (secon } \\
\text { ds) }\end{array}$ & 5.92 & $\begin{array}{l}0.6 \\
5\end{array}$ & $\begin{array}{l}7.9 \\
1\end{array}$ & $\begin{array}{l}0 . \\
64\end{array}$ & -1.99 & $\begin{array}{l}7.56 \\
7^{*}\end{array}$ \\
\hline \multirow[t]{2}{*}{ Balance } & $\begin{array}{l}\text { Stability } \\
\text { balance }\end{array}$ & $\begin{array}{l}\text { Standin } \\
\text { g with } \\
\text { feet on } \\
\text { bar } \\
\text { (secon } \\
\text { ds) }\end{array}$ & $\begin{array}{l}30.5 \\
6\end{array}$ & $\begin{array}{l}2.1 \\
5\end{array}$ & $\begin{array}{l}23 . \\
53\end{array}$ & $\begin{array}{l}2 . \\
64\end{array}$ & 7.03 & $\begin{array}{l}7.15 \\
2^{*}\end{array}$ \\
\hline & $\begin{array}{l}\text { Moving } \\
\text { balance }\end{array}$ & $\begin{array}{l}\text { walking } \\
\text { on } \\
\text { Swedis } \\
\text { h } \\
\text { bench } \\
\text { (secon } \\
\text { ds) }\end{array}$ & 5.49 & $\begin{array}{l}0.6 \\
2\end{array}$ & $\begin{array}{l}6.7 \\
4\end{array}$ & $\begin{array}{l}0 . \\
85\end{array}$ & -1.25 & $\begin{array}{l}4.11 \\
2^{*}\end{array}$ \\
\hline \multicolumn{2}{|l|}{ Accuracy } & $\begin{array}{l}\text { Shootin } \\
\text { g on }\end{array}$ & 6.14 & $\begin{array}{l}1.9 \\
3\end{array}$ & $\begin{array}{l}4.0 \\
2\end{array}$ & $\begin{array}{l}2 . \\
14\end{array}$ & 2.12 & $\begin{array}{l}2.54 \\
8^{*}\end{array}$ \\
\hline
\end{tabular}




\begin{tabular}{|c|c|c|c|c|c|c|c|}
\hline & $\begin{array}{l}\text { overlap } \\
\text { ped } \\
\text { rectang } \\
\text { les }\end{array}$ & & & & & & \\
\hline \multirow[t]{4}{*}{$\begin{array}{l}\text { Locomot } \\
\text { or test }\end{array}$} & $\begin{array}{l}\text { Time sense error } \\
10 \text { seconds } \\
\text { (second) }\end{array}$ & 1.71 & $\begin{array}{l}0.7 \\
2\end{array}$ & $\begin{array}{l}2.7 \\
5\end{array}$ & $\begin{array}{l}1 . \\
23\end{array}$ & -1.04 & $2.53^{*}$ \\
\hline & $\begin{array}{l}\text { Jump distance } \\
\text { sense error } 60 \mathrm{~cm} \\
(\mathrm{~cm})\end{array}$ & 3.36 & $\begin{array}{l}0.9 \\
5\end{array}$ & $\begin{array}{l}6.9 \\
3\end{array}$ & $\begin{array}{l}1 . \\
78\end{array}$ & -3.57 & $\begin{array}{l}6.13 \\
4^{*}\end{array}$ \\
\hline & $\begin{array}{l}\text { Compared visual } \\
\text { sense error for } \\
\text { walking distance } \\
10 \mathrm{~m}(\mathrm{~cm})\end{array}$ & $\begin{array}{l}37.6 \\
8\end{array}$ & $\begin{array}{l}1.5 \\
8\end{array}$ & $\begin{array}{l}43 . \\
23\end{array}$ & $\begin{array}{l}2 . \\
17\end{array}$ & -5.55 & $\begin{array}{l}7.16 \\
1^{*}\end{array}$ \\
\hline & $\begin{array}{l}\text { Compared visual } \\
\text { sense error for } \\
\text { walking direction } \\
10 \mathrm{~m}(\mathrm{~cm})\end{array}$ & $\begin{array}{l}23.2 \\
3\end{array}$ & $\begin{array}{l}2.3 \\
1\end{array}$ & $\begin{array}{l}31 . \\
73\end{array}$ & $\begin{array}{l}2 . \\
86\end{array}$ & -8.5 & $\begin{array}{l}8.01 \\
1^{*}\end{array}$ \\
\hline
\end{tabular}

* Significant at the 0.05 level $=2.074$

Figure (1) experimental and control groups means in locomotor and physical tests after experiment

Table (13) and figure (1) reveal significant differences between experimental and control groups post measurements in locomotor and physical tests as $\mathrm{T}$ value ranges between $(2.421$ and 12.67) values which is greater than T significant at 0.05 level.

Researcher attribute this improvement to the effectiveness of coordination exercises program, which applied to experimental group, with what it includes from varied and exciting gradually loaded exercises with and without tools, which led to improve 
some physical abilities (flexibility, speed, agility, coordination, balance, and accuracy). This effect reflected in improving locomotor abilities, so first hypothesis is accepted (There are statistically significant differences between pre and post measurements of some locomotor and physical abilities within experimental group handicapped children in favor of post measurements.).

Coordination has reciprocal relationships with physical and motor elements; as agility, accuracy, balance, speed and muscle ability are of the most important components of motor coordination. (Abbas, 2005, p. 103 and Ibrahim, 2007, p,82)

This coincides with what referred by Musa (2009, p.108) and Moghazi (2010, p.130) that mentally disabled children practicing of physical activities and games lead to motor abilities development (flexibility, speed, agility, coordination and balance). It also improve their motor performance and motor skills cognition to qualify them to do everyday life skills and self-reliance in face of daily life problems.

Due to delayed motor development for mentally disabled children and what they face from dispersion and disorders, they are less capable of walking and running in right way, and they are behind at least two years from normal child. They reach their complete ability in muscle coordination, motor skills, body balance and locomotor cognition physical and perceptual only by organized physical education programs in home and at school. (Moghazi, 2010, p.120)

This is confirmed by Taha (2006, p.16), where he stressed on that physical training for handicapped children (mental disability) make progress in their motor performance and help them to learn mental cognitive skills. This because mentally disabled child delayed in sitting, standing, walking, jumping and running, so he needs to exercise for development of motor abilities in general and motor balance in particular.

Improvement in locomotor abilities is due to gradual education, using visual stimuli to help develop visual and motor sense, giving a model for proper performance of each movement and correct errors during performance, and regular training that improves the functions of motor analyzers. In addition, improving physical abilities lead to development of locomotor perception (Shaaban, 2012, p.88)

Control group progress in locomotor and physical abilities, even it is simple and non-remarkable, is due to the nature of school motor activity, as constancy in training, physical exercise and 
motor activities lead to improve physical ability and locomotor perception. (Shaaban, 2012, p.14 and Gouda , 2014, p.102)

Table (14) Significance of differences between experimental and control groups in ADHD checklist after experiment

\begin{tabular}{l|l|l|l|l|l|l}
\multicolumn{1}{r}{$\begin{array}{r}\text { Statistical } \\
\text { indicators } \\
\text { variables }\end{array}$} & \multicolumn{2}{l|}{$\begin{array}{l}\text { Experimental } \\
\text { group (n=12) }\end{array}$} & \multicolumn{2}{|c|}{$\begin{array}{c}\text { Control } \\
\text { group } \\
(\mathbf{n = 1 2 )}\end{array}$} & $\begin{array}{l}\text { Mean } \\
\text { difference }\end{array}$ & \\
\cline { 2 - 6 } & Mean & $\pm S D$ & Mean & $\pm S D$ & & \\
\hline Attention Deficit & 37.19 & 3.76 & 46.36 & 5.12 & -9.17 & $5.000^{*}$ \\
\hline Hyperactivity & 35.67 & 3.35 & 48.86 & 4.75 & -13.19 & $7.861^{*}$ \\
\hline Impulsivity & 29.36 & 2.95 & 44.57 & 4.67 & -15.21 & $9.536^{*}$ \\
\hline Total & 102.22 & 7.68 & 139.79 & 8.42 & -37.57 & $11.419^{*}$ \\
\hline
\end{tabular}

* Significant at the 0.05 level $=2.074$

Table (14) and figure (2) reveal significant differences between experimental and control groups post measurements in ADHD checklist as $T$ value ranges between (5.000 and 11.419) values which is greater than $T$ significant at 0.05 level.

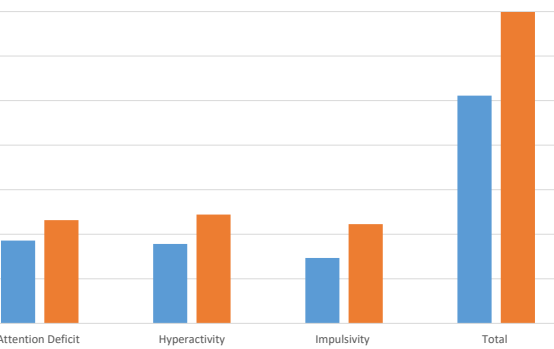

Figure (2) experimental and control groups means in ADHD checklist after experiment

Researcher attribute this improvement to the effectiveness of the proposed physical exercise program which was effective on easing ADHD in mentally disabled children, so second hypothesis is accepted (There are statistically significant differences between post measurements of some locomotor and physical abilities within experimental and control group handicapped children in favor of experimental group)

This confirmed Alsharif (2014) study results, which proofed that recreation programs using exercise with tools was effective in easing ADHD within learnable mentally disabled children.

Mentally disabled child participation in exercises and motor activities help him to achieve rehabilitative goals not only in physical and motor aspects, but also in psychological aspects, ease behavioral and social disorders, and the acquisition of 
healthy behaviors and habits through social interaction among participants. (Kashef, 2002, p. 45; Ben Gawad \& Hassan, 2005, p34; Abdulgawad, 2009, p.89; Ghorab, 2010, p. 32; Hassn, 2011, p.65; and Abdulaziz, 2013, p.67)

This is confirmed by study results of Abuzaid (2004), Morsi (2006), Briere, D. E., III, \& Siegle (2008), and Alsharif (2014), which confirmed that public attitudes towards sports activities within handicapped children are more positive to achieve best level in easing behavioral disorders, and to get rid of negative and impulsive emotions.

\section{Conclusions:}

1- Using coordination exercises led to improve locomotor and physical abilities within handicapped children

2- Using coordination exercises led to ease ADHD within handicapped learnable children

3- Using coordination exercises is better than using motor activities normally used in handicapped children schools

\section{Recommendations:}

1- using ADHD observation checklist in establishments that care with learnable handicapped children.

2- Applying the coordination exercises program in these establishments with help of physical education graduates.

3- It is necessary for intellectual schools program planners to get interest with physical abilities development program and implement it

4- Provide assisting tools that used in physical education classes in intellectual education schools.

5- There is a need for further scientific studies of this age group.

\section{References}

Salama, I. A. (2000). Applied approach of measurement in physical fitness, Alexandria: Dar EL-Maaref. (In Arabic Language)

Abdulfatah, A. A. (1997). Sport training and physiological basis. Cairo, Egypt: Arab thought house.

Alsharif, A. M. (2014). Effect of recreational program using tools in reducing attention deficit accompany hyperactivity within learn-able mentally retarded children (Unpublished master's thesis). Faculty of physical education for girls, Alexandria, Egypt.

Morsi, A. M. (2006). Effect of exercises program using tools with merging and isolating systems on social interaction and separation anxiety within Down syndrome learn-able 
children. Sports sciences and arts, 25(3). Faculty of physical education for girls, helwan university

Abdulaziz, A. G. (2013). Effect of emotive intelligence development program on reducing attention deficit accompany hyperactivity disorder within primary school pupils (Unpublished doctoral dissertation). Faculty of education, Suez University, Egypt.

Alhagrasy, A. M. (2002). Educating mentally retarded children. Cairo: Arab Thought house.

Kashef, I. M. (2002). Integrating handicapped with normal children. Cairo: Modern book house.

Ahmed, B. M. (1999). Cairo, Egypt: Arab thought house.

Taha, G. S. (2006). Proposed small games program and its effect on improving some basic motor skills [performance and social behavior within mentally handicapped pupils(Unpublished master's thesis). Faculty of Physical education, Alexandria University, Egypt.

Hassn, T. S. (2011). Effectiveness of a program to develop self organization ability on reducing attention deficit hyperactivity disorder within primary school students (Unpublished doctoral dissertation). Faculty of education, Beni suef University, Beni Suef, Egypt.

Essawi, J. A (1999). Effect if using ball to acquire neuromuscular coordination on some upper limb muscles electrical activity within rhythmic gymnastics juniors (Unpublished master's thesis). Faculty of physical education for girls, Helwan university, Cairo, Egypt.

Nawasra, H. M. (2010). Handicapped, introduction to physical rehabilitation. Cairo, Egypt: Collegiate press house.

Alqady, K. S. (2011). behavior amendment of attention deficit hyperactivity disorder children. Cairo, Egypt: Book world.

Abdulgawad, R. M. (2009). Attention deficit hyperactivity disorder and its relation with social and emotion learning difficulties within late childhood children (Unpublished master's thesis). Faculty pf arts, Menia University, Menia, Egypt.

Alkhashrany, S. A., \& EsSayed, E. A. (2009). Attention deficit hyperactivity disorder scale for children (validation study). Arab special education journal, special education academy, Riyadh, (14).

Shaaban, S. A. (2013). the effect of specific exercise on dynamics development of Kinaesthetic Perception of some motor skills for gymnastics of all players, theories and applications, specialized journal in Physical Education and Sports 
Science. Issue 79. Faculty of Physical Education for men, Alexandria University. (In Arabic Language)

Abdullah, A. (2003). Behavior adjustment for mentally retarded children using pictured activity tables, applied studies. Cairo, Egypt: Rashad House.

Ben Gawad, A., \& Hassan, A. A. (2005). Physical education for handicapped. Dubai, UAE: Learning house for publishing and distribution.

Musa, A. S. (2009) .Effect of introductory games program on some football motor abilities and basic skills within intellectual schools pupils (Unpublished master's thesis). Faculty pf physical education for men, Alexandria university, Aleandria, Egypt.

Abdul Khaliq, E.M. (2005), sports training, theories and applications. $12^{\text {th }}$ ed. Alexandria: Knowledge House. (In Arabic)

Abbas, E. (2005). planning and scientific bases for building teams in group games. Alexandria, Egypt: Maaref establishment.

Ibrahim, F. A. (2005). Scientific principles and basis of physical exercises and mass artistic performances. Aleandria, Egypt: Waffaa publishing house.

Macintyre, C. (2002). Play for children with special needs: Including children aged 3-8 (K. Alamry, Trans.). Cairo, Egypt: Farouq house.

Gouda, L. M(2014). .Effect of specific purpose exercises on development dynamic of some locomotor cognition variables and improving performance level within under 15 years gymnastic for all female players (Unpublished master's thesis). Faculty of physical education for men, Alexandria University, Alexandria, Egypt.

Shamoon, M. A. (2002). Sport psychology and psychological measurement. Cairo: Book publishing center. (In Arabic Language)

Hassanien, M. S. (2001). Measurement and evaluation in physical education (4th ed.). Cairo, Egypt: Arab thought house.

Ibrahim, M. A.(2007). Electromechanical indicators as a basis for developing neuromuscular coordination in foil fencing 1-2 attack (Unpublished master's thesis). Faculty of physical education for men, Zagazig University, Zagazig, Egypt.

Mahmoud, M. A., \& Qandil, M. (2005). Physical education for basic education. Mansoura, Egypt: National book house.

Aldesouqy, M. M. (2006). Attention deficit hyperactivity disorder. Cairo, Egypt: Egyptian Anglo. 
Mohammad, M. A. (2010). Attention deficit hyperactivity disorder scale within autism children (9-12). Amman, Jordan: Sfaaa house for publishing and distribution.

Allawi, M.H. (1994), sports training science. $13^{\text {th }}$ ed., Cairo: Knowledge House, (In Arabic)

Moghazi, M. A(2010). .Effect of developing some specific physical abilities on performing some basic motor skills within mentally retarded aged 8-12 years (Unpublished master's thesis). Faculty o physical education fro men, Alexandria University, Alexandria, Egypt.

Abuzaid, M. M. (2004). Effectiveness of proposed small games program on improving life adjustment within mentally retarded (Unpublished master's thesis). Faculty of physical education for girls, Alexandria University, Alexandria, Egypt.

Ghorab, H. A. (2010). Guiding program to reduce attention deficit hyperactivity disorder within children with learning difficulties. Arabic Education future, Faculty of education, Cairo University, 17(63), 345-422.

Special education general management, basic education general management, high education sector, Ministry of Education. (2003). Administration instruction and technical guides for intellectual schools. Cairo, Egypt: Author.

American Psychiatric Association. (2000). Diagnostic and statistical manual of mental disorders: DSM-IV-TR. Washington, DC: Author.

Barkley, R. A. (2003). Issues in the diagnosis of attentiondeficit/hyperactivity disorder in children. Brain and Development, 25(2), 77-83. doi:10.1016/s03877604(02)00152-3

Briere, D. E., III, \& Siegle, D. (2008). The effects of the Unified Sports basketball program on special education students' self-concepts: Four students' experiences. TEACHING Exceptional Children Plus, 5(1) Article 1. Retrieved [Nov 2015] from http://escholarship.bc.edu/education/tecplus/vol5/iss1/art1

ALhussainy, L. (2010, April 11) .Godant-Haris scale .Retrieved from http://www.acofps.com/vb/showthread.php?t=5872-39. Accessed September 2015 
Physical tests

Appendix (1)

\begin{tabular}{|c|c|c|c|c|}
\hline \multicolumn{2}{|c|}{ Physical abilities } & Test & $\begin{array}{c}\text { Measuremen } \\
\mathbf{t} \\
\text { unit }\end{array}$ & Reference \\
\hline \multicolumn{2}{|l|}{ Flexibility } & $\begin{array}{l}\text { Trunk } \\
\text { front } \\
\text { flexion } \\
\text { from long } \\
\text { sitting test } \\
\end{array}$ & $(\mathrm{cm})$ & $\begin{array}{l}\text { (Hassanien } \\
\text {, 2001, p, } \\
65)\end{array}$ \\
\hline \multicolumn{2}{|l|}{ speed } & $\begin{array}{l}20 \mathrm{~m} \\
\text { running }\end{array}$ & (second) & $\begin{array}{l}\text { (Hassanien } \\
\text { 2001, p, } \\
89)\end{array}$ \\
\hline \multicolumn{2}{|l|}{ agility } & \begin{tabular}{|l|} 
Zigzag \\
running
\end{tabular} & (seconds) & $\begin{array}{l}\text { (Hassanien } \\
\text {,2001, p, } \\
357) \\
\end{array}$ \\
\hline \multirow[t]{2}{*}{$\begin{array}{l}\text { coordinatio } \\
\text { n }\end{array}$} & $\begin{array}{l}\text { Eye-hand } \\
\text { coordinatio } \\
n\end{array}$ & $\begin{array}{l}\text { Ball throw } \\
\text { and } \\
\text { receive } \\
\text { within } 30 \\
\text { seconds }\end{array}$ & (number) & $\begin{array}{l}\text { (Hassanien } \\
\text { 2001, p, } \\
410)\end{array}$ \\
\hline & $\begin{array}{l}\text { Eye-legs } \\
\text { coordinatio } \\
\mathrm{n}\end{array}$ & $\begin{array}{l}\text { Numbered } \\
\text { circles test }\end{array}$ & (seconds) & $\begin{array}{l}\text { (Hassanien } \\
\text { 2001, p, } \\
412)\end{array}$ \\
\hline \multirow[t]{2}{*}{ Balance } & $\begin{array}{l}\text { Stability } \\
\text { balance }\end{array}$ & $\begin{array}{l}\text { Standing } \\
\text { with feet } \\
\text { on bar }\end{array}$ & (seconds) & $\begin{array}{l}\text { (Hassanien } \\
\text { 2001, p, } \\
434)\end{array}$ \\
\hline & $\begin{array}{l}\text { Moving } \\
\text { balance }\end{array}$ & $\begin{array}{l}\text { walking on } \\
\text { Swedish } \\
\text { bench }\end{array}$ & (seconds) & $\begin{array}{l}\text { (Hassanien } \\
\text { 2001, p, } \\
429)\end{array}$ \\
\hline \multicolumn{2}{|l|}{ Accuracy } & $\begin{array}{l}\text { Shooting } \\
\text { on } \\
\text { overlappe } \\
\text { d } \\
\text { rectangles }\end{array}$ & (number) & $\begin{array}{l}\text { (Hassanien } \\
\text { 2001, p, } \\
441)\end{array}$ \\
\hline
\end{tabular}

Locomotor tests:

\begin{tabular}{c|c|c}
\hline Test & $\begin{array}{c}\text { Measurement } \\
\text { unit }\end{array}$ & Reference \\
\hline Time sense error 10 seconds & (seconds) & (Salama, \\
\hline
\end{tabular}




\begin{tabular}{l|r|r}
\hline & & $2000, \mathrm{p}$, \\
& & $168)$ \\
& & Shamoon, \\
& $2002, \mathrm{p}$, \\
& & $214)$ \\
\hline Jump distance sense error $60 \mathrm{~cm})$ & $(\mathrm{cm})$ & $($ Salama, \\
& & $2000, \mathrm{p}$, \\
& & $164)$ \\
& & Shamoon, \\
wompared visual sense error for & $(\mathrm{cm})$ & $($ Salama, \\
walking distance 10m & & $2002, \mathrm{p}$, \\
& & $165)$ \\
\hline Compared visual sense error for & $(\mathrm{cm})$ & $($ Shamoon, \\
walking direction 10m & & $2002, \mathrm{p}$, \\
& & $215)$ \\
\hline
\end{tabular}




\section{Appendix (2)}

ADHD checklist from DSM IV, amended by Alsharif (2014), it made up of three aspects containing (32) phrases on quintuple response scale (always happen, often happen, sometimes happen, rarely happen, never happen) with scores $(5,4,3,2,1)$ respectively.

First aspect: Attention deficit: phrases 1, 4, 7, 10, 13, 16, 19, 22, 25, 28, 31.

Second aspect: Hyper activity: phrases 2, 5, 8, 11, 14, 17, 20, 23, 26, 29, 32

Third aspect: Impulsivity: phrases 3, 6, 9. 12, 15, 18, 21, 24, 27, 30.

\begin{tabular}{|c|c|c|c|c|c|c|}
\hline $\begin{array}{l}N \\
0\end{array}$ & Phrases & $\begin{array}{l}\text { Always } \\
\text { happen }\end{array}$ & $\begin{array}{l}\text { Often } \\
\text { happe } \\
\mathrm{n}\end{array}$ & $\begin{array}{l}\text { Sometime } \\
\text { s } \\
\text { happen }\end{array}$ & $\begin{array}{l}\text { Rarely } \\
\text { happe } \\
\mathrm{n}\end{array}$ & $\begin{array}{l}\text { Never } \\
\text { happe } \\
n\end{array}$ \\
\hline 1 & $\begin{array}{l}\text { Easily } \\
\text { Distracted }\end{array}$ & & & & & \\
\hline 2 & $\begin{array}{l}\text { fails to give } \\
\text { close } \\
\text { attention to } \\
\text { details }\end{array}$ & & & & & \\
\hline 3 & $\begin{array}{l}\text { makes } \\
\text { careless } \\
\text { mistakes in } \\
\text { schoolwork }\end{array}$ & & & & & \\
\hline 4 & $\begin{array}{l}\text { has difficulty } \\
\text { sustaining } \\
\text { attention in } \\
\text { tasks or } \\
\text { activities }\end{array}$ & & & & & \\
\hline 5 & $\begin{array}{l}\text { Bored by } \\
\text { performance } \\
\text { one activity } \\
\text { after few } \\
\text { minutes and } \\
\text { move from } \\
\text { one activity } \\
\text { to another }\end{array}$ & & & & & \\
\hline 6 & $\begin{array}{l}\text { fails to finish } \\
\text { school work } \\
\text { and things } \\
\text { he started }\end{array}$ & & & & & \\
\hline 7 & $\begin{array}{l}\text { loses things } \\
\text { necessary }\end{array}$ & & & & & \\
\hline
\end{tabular}




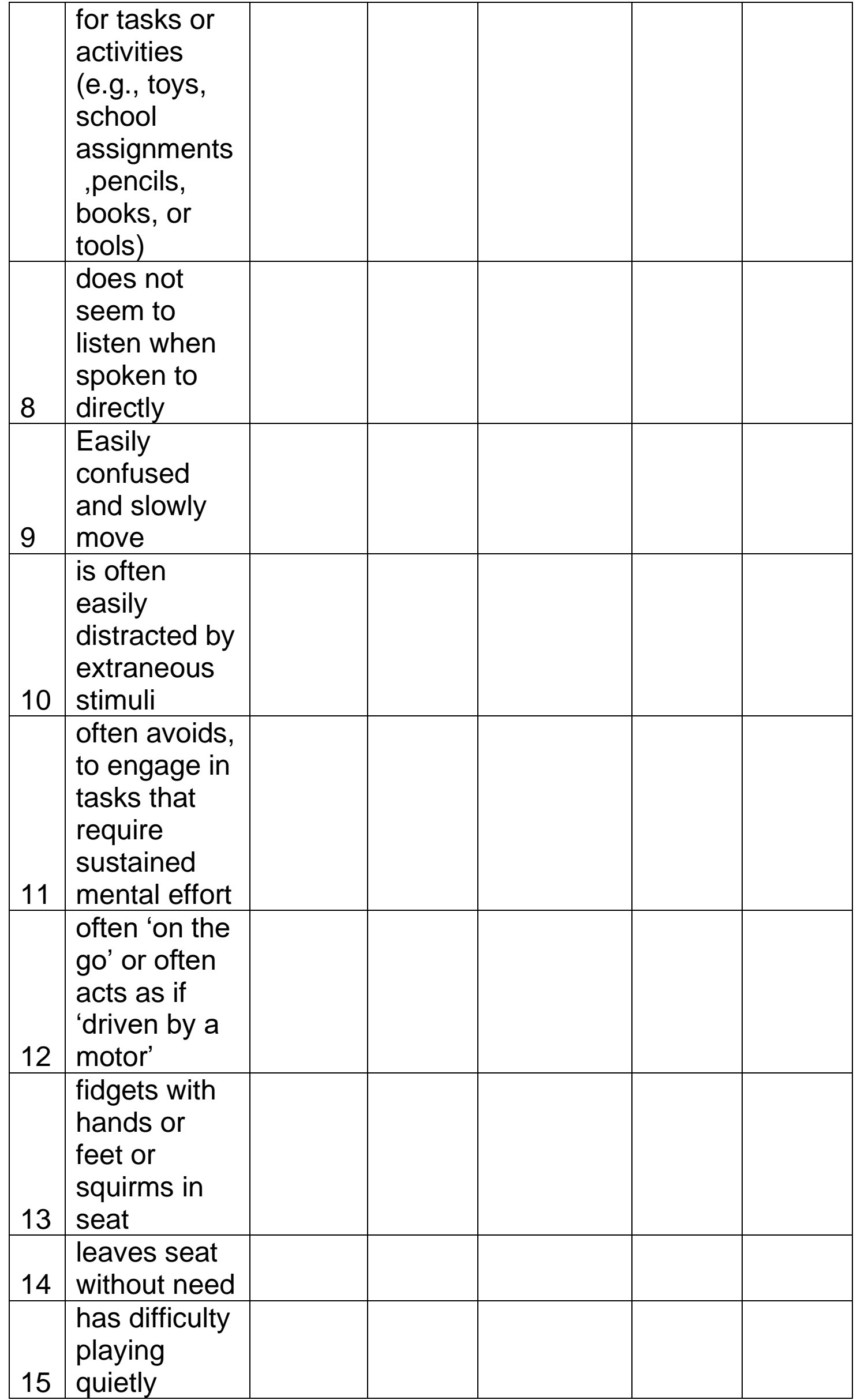




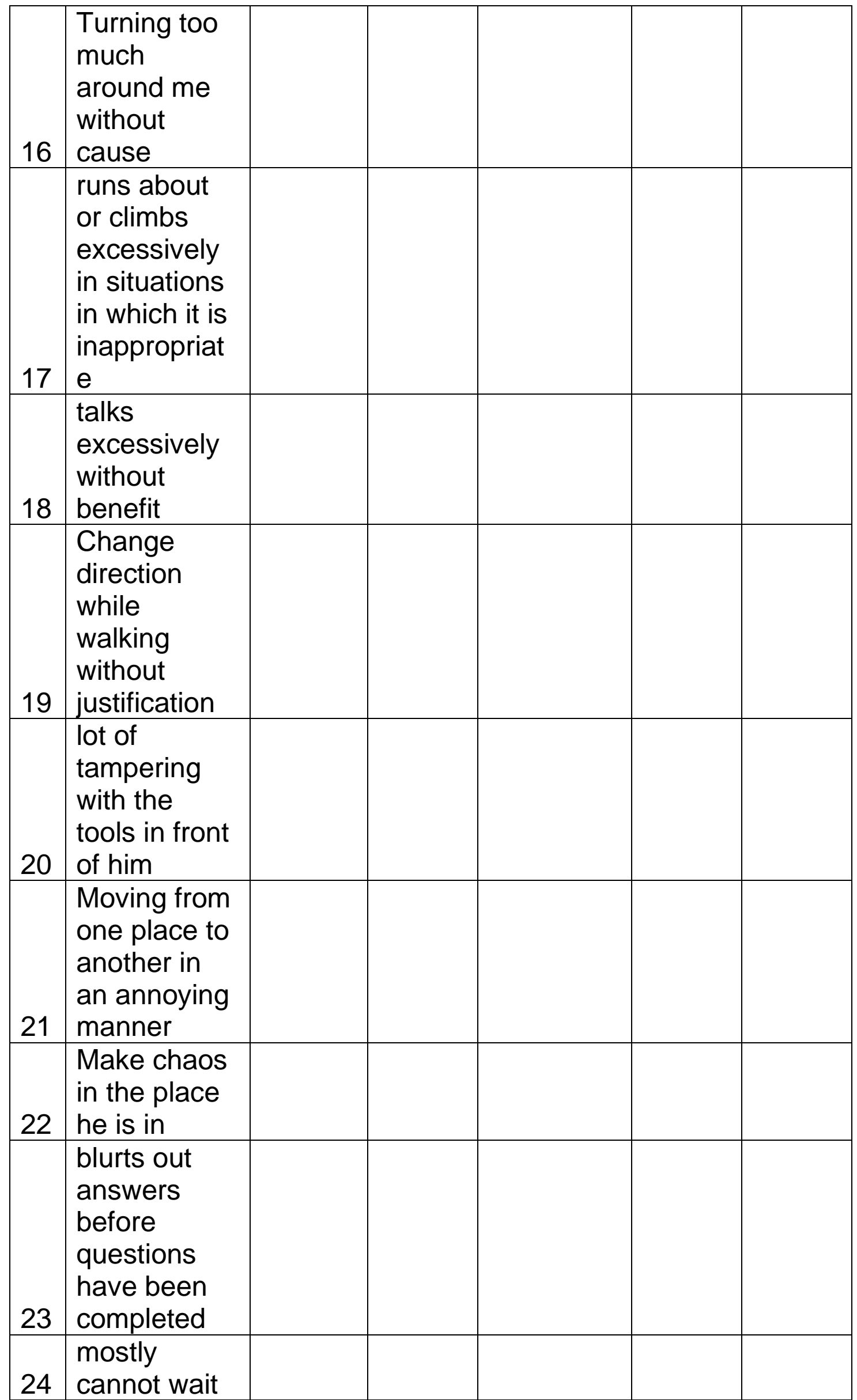




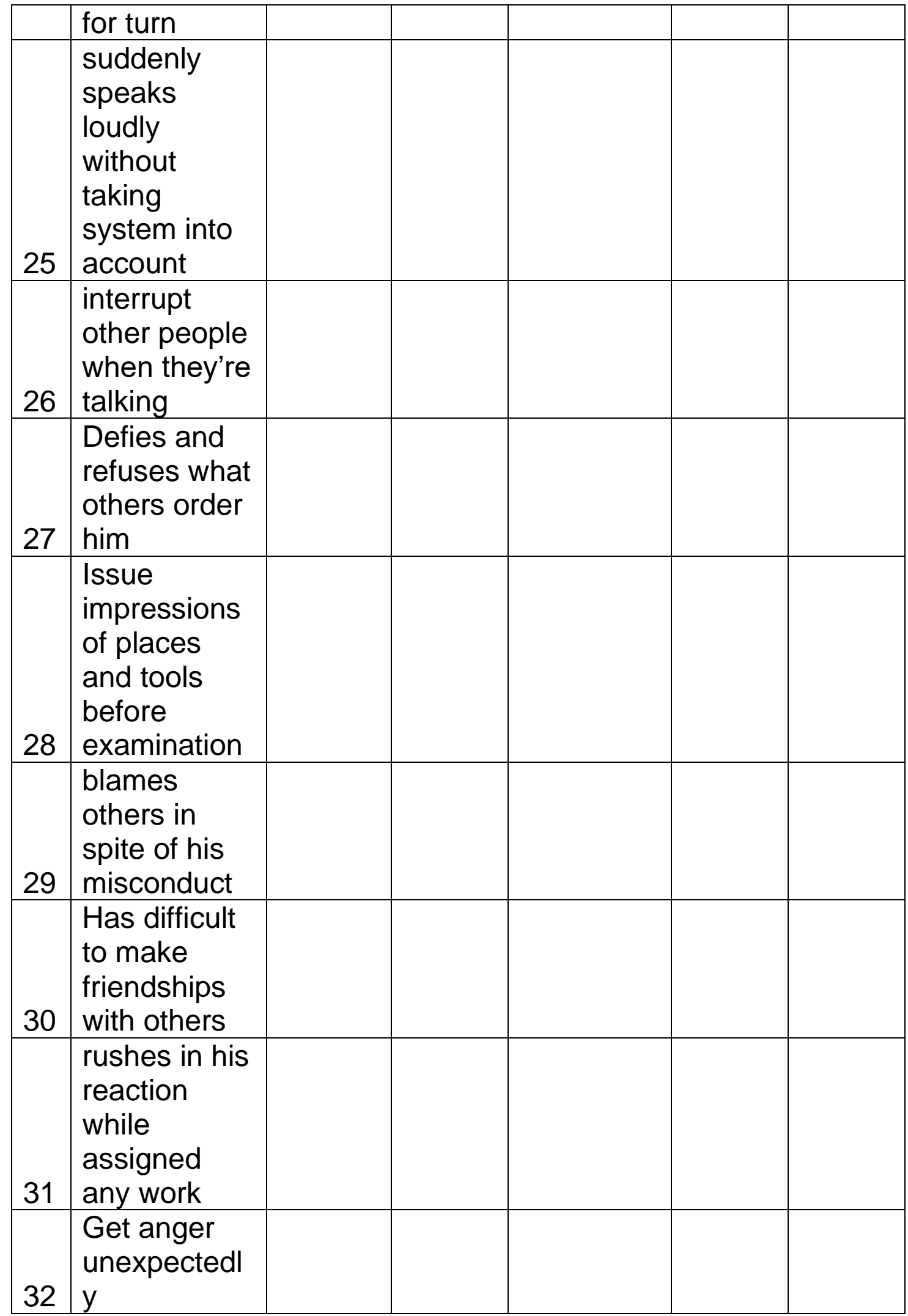

\section{ADHD checklist validity}

Internal consistency coefficient between aspects and over all list ranged between (0.941 and 0.989), the matter confirms aspects validity, Internal consistency coefficient between aspects ranged between (0.869 and 0.973), the matter confirms that aspects are 
significantly correlated, and measure what checklist measure and characterized with self validity.

\section{ADHD Reliability:}

Alpha Cronbach reliability coefficient for checklist phrases ranged between $(0.675$ and 0.746$)$ and for the list was $(0.881)$, the matter that confirm aspects homogeneity; and that it characterized with reliability. Test/retest reliability coefficient ranged between (0.904 and 0.996$)$ the matter which confirms variables reliability. 